\title{
Prolonged QT interval in Rett syndrome
}

\author{
C J Ellaway, G Sholler, H Leonard, J Christodoulou
}

\begin{abstract}
Rett syndrome is a severe neurodevelopmental disorder of unknown aetiology. A prolonged QT interval has been described previously in patients with Rett syndrome. To investigate QT prolongation and the presence of cardiac tachyarrhythmias in Rett syndrome electrocardiography and 24 hour Holter monitoring were performed prospectively in a cohort of 34 girls with Rett syndrome. The corrected QT value was prolonged in nine patients. Compared with a group of healthy controls of a similar age range, the patients with Rett syndrome had significantly longer corrected QT values. Clinical severity was not a predictor for prolonged QT intervals in the Rett syndrome cohort. The prolonged QT syndrome is a serious and potentially lethal cardiac disorder and should be considered in all girls with Rett syndrome.

(Arch Dis Child 1999;80:470-472)
\end{abstract}

Keywords: Rett syndrome; prolonged QT; electrocardiography

Rett syndrome is a severe neurodevelopmental disorder occurring almost exclusively in girls/ women. It is characterised by normal early development, followed by developmental regression with loss of communication skills, deceleration of head growth, gait ataxia, loss of purposeful hand function, and the development of stereotypic hand movements. ${ }^{1}$ Prolonged rate corrected QT intervals (QTc) have been identified previously in patients with Rett syndrome. ${ }^{2}$ We report the results of prospective electrocardiogram (ECG) and 24 hour Holter monitor studies in a cohort of 34 patients with Rett syndrome. To determine whether the QTc values found in our Rett syndrome cohort are significantly longer than normal we compared the results with a group of healthy control girls of a similar age range. Furthermore, we correlate the findings with the clinical severity of Rett syndrome.

\section{Methods}

We recruited 34 patients from the Australian Rett Syndrome Register as part of a wider clinical review. The register, a database of known Rett syndrome cases, was established during the years 1993 to 1995 through active ascertainment by the Australian Paediatric Surveillance Unit and other sources of notification, such as the Rett Syndrome Association of Australia. ${ }^{3}$ At the time of commencement of our study there were 39 known patients with Rett syndrome under the age of 20 years in New South Wales. The diagnosis of Rett syndrome was confirmed by one of us (CE): patients were classified as having classic Rett syndrome or an atypical variant and the clinical stage determined using established diagnostic and staging criteria. ${ }^{4-6}$ Clinical information was collected regarding the presence of a seizure disorder, medications, and mobility status. Blood was collected for plasma calcium, potassium, and magnesium.

We performed a standard 12 lead ECG and a 24 hour Holter monitor on all 34 patients with Rett syndrome at The Royal Alexandra Hospital for Children. An automated calculation of the QTc value was made and rechecked by a single paediatric cardiologist (GS). The QT interval, determined by the longest hand measured QT interval in any lead, was corrected for the heart rate by the method of Bazett to yield the QTc value. ${ }^{7}$ We considered the QTc to be normal if less than 0.45 seconds and borderline if between 0.44 and 0.45 seconds. All ECGs and Holter monitor recordings were reviewed by the same paediatric cardiologist (GS). The comparison population consisted of 417 female subjects aged 3-20 years with no cardiac abnormality, in whom electrocardiography and echocardiography had been performed for routine screening of a murmur.

We used analysis of variance to test for between group differences of the Rett syndrome cohort and controls after adjusting for age as a covariate. We determined the mean QTc values and standard errors for each group and the clinical stage of the Rett syndrome cohort. Scatterplots with regression lines of best fit were created for the Rett syndrome cohort and the control group. We analysed QTc values across clinical stage by the Kruskal-Wallis test. We used $\chi^{2}$ analysis to test groups categorised for age $>12$ years, mobility status, type of Rett syndrome, and the presence of a seizure disorder. We used Fisher's exact test for tables with an expected cell count of less than five. Statistical analyses were performed using the SPSS statistics package, version 6.1.

\section{Results}

Of the 34 patients, 30 had classic Rett syndrome and four were classified as atypical Rett syndrome. Ages ranged from 3.5 to 20 years, with a median age of 9 years. Twenty three patients were aged less than 12 years. Excluding four cases recruited from interstate, our cohort represented about three quarters of the juvenile cases of Rett syndrome in New South Wales.

Four patients were in clinical stage II, 26 in clinical stage III, and four in clinical stage IV of the disorder. Nineteen patients were able to 


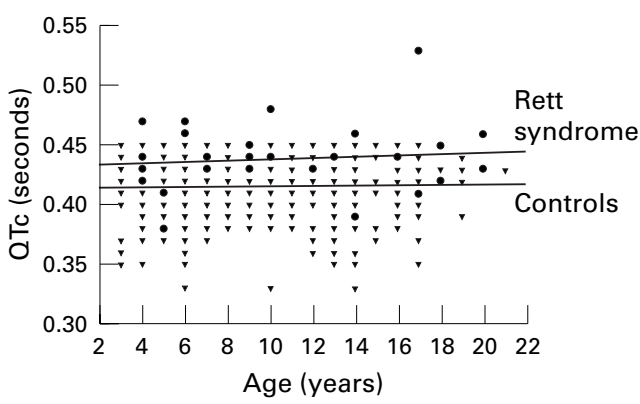

Figure 1 Scatter plot with regression line of best fit for the Rett syndrome cohort and controls. Closed circles, patients with Rett syndrome; closed triangles, controls.

walk; 21 had a history of seizures, of whom 16 were taking anticonvulsants-five were taking lamotrigine, nine carbamazepine, six sodium valproate, one phenytoin, three clobazam, and one diazepam. Seven patients were taking more than one anticonvulsant at the time of the ECG.

The QTc values of the Rett syndrome cohort ranged from 0.38 to 0.53 seconds with a mean of 0.44 seconds. A prolonged QTc $(\geqslant 0.45$ seconds) was identified in nine patients (confidence interval (CI), 11.7 to 41.3). The corrected QT interval in these patients ranged from 0.45 to 0.53 seconds, with a mean of 0.47 seconds. A total of 26 patients was assigned to stage III Rett syndrome, of whom eight were found to have a prolonged QTc value. One of four patients in stage II and one of four in stage IV had QTc prolongation. Ten patients had a borderline QTc value of 0.44 seconds, of whom one was in clinical stage II, seven were in clinical stage III, and one in clinical stage IV of the disorder. Plasma potassium, calcium, and magnesium levels were within normal limits for all patients. Six of the patients with a prolonged QTC were not taking any medications.

The QTc values of the normal controls ranged from $0.33-0.45$ seconds with a mean of 0.42 seconds. Age adjusted comparison between the Rett syndrome cohort and the control group by analysis of variance demonstrated a significant between group difference: variance ratio $(F)=28.5$; degrees of freedom $(d f)=1,450 ; p<0.001$. Figure 1 shows the

Table 1 Corrected $Q T(Q T c)$ values of the control group and the Rett syndrome cohort together with the clinical stage of Rett syndrome

\begin{tabular}{lclll}
\hline & Number & Mean QTc (seconds) & Standard error & p Value \\
\hline Control group & 417 & 0.415 & 0.001 & \\
Rett syndrome cohort & 34 & 0.438 & 0.004 & $\mathrm{p}<0.001^{\star}$ \\
Clinical stage & 4 & 0.410 & 0.042 & \\
$\quad$ II & 26 & 0.441 & 0.027 & \\
III & 4 & 0.437 & 0.008 & $\mathrm{p}=0.7 \dagger$ \\
IV & &
\end{tabular}

${ }^{\star}$ Calculated by analysis of variance for between group difference adjusted for age; $†$ for $Q T c$ across clinical stage of Rett syndrome calculated by Kruskal-Wallis test.

Table 2 Proportion of Rett syndrome cohort categorised according to age and clinical characteristics with prolonged QTC

\begin{tabular}{lllll}
\hline Patient characteristic & Prolonged QTc & p Value & Odds ratio & $95 \%$ CI \\
\hline Age > 12 years & $3 / 11$ & 1.0 & 1.0 & 0.4 to 3.0 \\
Classic Rett syndrome & $9 / 30$ & 0.6 & 1.2 & 1.0 to 1.4 \\
Seizure disorder & $4 / 21$ & 0.3 & 0.7 & 0.3 to 1.4 \\
Lack of mobility & $2 / 15$ & 0.2 & 0.4 & 0.1 to 1.5 \\
\hline
\end{tabular}

CI, confidence intervals. scatterplots with regression lines of best fit for the patients with Rett syndrome and the control group.

Table 1 shows the mean QTc value and standard error of the control group and the Rett syndrome cohort in addition to the mean for each clinical stage of Rett syndrome. The QTc values were not significantly prolonged across clinical stages in patients with Rett syndrome $(\mathrm{p}=0.7)$.

Table 2 shows the results of $\chi^{2}$ analysis with Fisher's exact two tailed test. These results show that classic Rett syndrome $(\mathrm{p}=0.6)$, age $>12$ years $(p=1.0)$, the presence of a seizure disorder $(p=0.3)$, and lack of mobility $(\mathrm{p}=0.2)$ were not associated with QTc prolongation.

The 24 hour Holter monitor studies of the patients with Rett syndrome revealed no tachyarrhythmia, although one patient had a period of junctional escape bradycardia of 27 beats/ min during sleep. This patient had a QTc of 0.46 seconds. Sinus bradycardia was demonstrated in one patient who had a QTc of 0.46 seconds.

\section{Discussion}

Rett syndrome is a severe neurodevelopmental disorder of unknown aetiology occurring almost exclusively in girls/women. Affected girls are considered developmentally normal for the first 6 to 18 months, although subtle signs may already be present in the first 6 months. ${ }^{8}$ This is followed by a period of developmental stagnation then regression and social withdrawal. These girls lose functional hand skills and the use of spoken language and develop characteristic hand stereotypes and gait and/or truncal ataxia. Growth is characterised by early deceleration of head growth, leading to microcephaly in some cases, and a subsequent deceleration in linear growth, leading to short stature by later childhood. ${ }^{1}$ Diagnosis is based on a consistent constellation of clinical features and the utilisation of established diagnostic criteria. ${ }^{4}$ The natural history of the disorder has been divided into four clinical stages. ${ }^{6}$ Survival into adulthood is not unusual, although sudden deaths have been reported. Longitudinal records of Rett syndrome cases in the UK report an annual mortality rate of $1.2 \%$, with $26 \%$ of the deaths being sudden and unexpected. ${ }^{9}$

A prolonged QTc value has been reported previously in Rett syndrome. Sekul et al reported QTc prolongation (QTc $\geqslant 0.45$ seconds) in $41 \%$ of their cohort, with an increase in the mean value across clinical stages. The authors postulated that a prolonged QTc interval could be a possible cause of sudden death in Rett syndrome. ${ }^{2}$ Using the same criteria as Sekul et al, we found a significant difference in the QTc values between our Rett syndrome cohort and normal controls. However, we found no evidence of T or $\mathrm{U}$ wave abnormalities in our patients with Rett syndrome. Follow up of our cohort with 24 hour Holter monitors demonstrated no significant arrhythmias. Our statistical analyses did not reveal any risk factors for the presence of a 
prolonged QTc in Rett syndrome including the presence of a seizure disorder, clinical stage, age, lack of mobility, or medications. Although no correlation was identified between the presence of a prolonged QTc and clinical phenotype, these findings may ultimately provide positive information regarding the potential genetic heterogeneity of Rett syndrome.

The prolonged QT syndrome is a serious and potentially lethal cardiac disorder. The condition is believed to be caused by abnormal repolarisation after ventricular systole, and has been attributed to abnormality at the myocardial cellular level. The clinical features of abrupt loss of consciousness, seizures, or sudden death result from episodic malignant ventricular arrhythmias; specifically, repolarisation related ventricular tachyarrhythmias, such as torsade de pointes and ventricular fibrillation. ${ }^{10}$ The risk of sudden death can be significantly reduced in such patients by the use of $\beta$ blockers. Acquired long QTc and secondary arrhythmias can result from cardiac ischaemia, hypothermia, and electrolyte disturbances such as hypokalaemia, hypocalcaemia, and hypomagnesaemia. They can also be associated with disorders of the central nervous system (CNS) including head injury, cerebrovascular accidents, and neurosurgical procedures, and can occur as an idiosyncratic response to drugs including antiarrhythmics, certain antibiotics, anaesthetic agents, and cisapride. ${ }^{11}$ Inherited forms of long QT syndrome can result from mutations in at least five different genes. ${ }^{12}{ }^{13}$ The most common cause of inherited long QT is mutation of the LQT1 gene, which has been mapped to chromosome $11 \mathrm{p} 15.5$. It encodes the $\alpha$ subunit of the $\mathrm{I}_{\mathrm{Ks}}$ potassium channel. Four other gene loci for inherited long QT syndromes have been identified. LQT2 or HERG, on chromosome 7q35-36 encodes the $I_{\mathrm{Kr}}$ potassium channel. LQT3 or SCN5A on chromosome 3p21-24 encodes a sodium channel, and the fourth locus LQT4, is on chromosome 4q25-27. The fifth locus LQT5 or KCNE1 on chromosome 21 encodes the $\beta$ subunit of the $I_{\mathrm{Ks}}$ potassium channel. Mutations in the gene for LQT1 are responsible for more than $50 \%$ of inherited long QT. ${ }^{6}$ Although the underlying genetic basis for Rett syndrome is not known, there is increasing evidence suggesting that the gene responsible lies on the $\mathrm{X}$ chromosome, ${ }^{7}$ although an autosomal locus has not been excluded. It is not clear whether a prolonged QTc interval in Rett syndrome is a direct effect of Rett syndrome on the heart or a secondary effect of the CNS manifestations of Rett syndrome. Because age and clinical status were not found to be independent influences on QTc prolongation in our cohort, we speculate that a secondary effect is less likely and a shared or linked cellular defect may be the underlying cause of QTc prolongation in Rett syndrome.

Based on our data, our current approach is to perform an ECG on all patients with Rett syndrome to identify the presence of QTc prolongation. In those who are found to have a prolonged QTc we then perform a 24 hour Holter monitor to screen for cardiac tachyarrhythmias. We recommend caution in diagnosing epilepsy in these individuals until prolonged QTc and associated arrhythmias have been ruled out. The decision regarding treatment for long QTc should then be discussed with the family, and is based on the patient's clinical condition, degree of QTc prolongation, and Holter results. We have chosen to monitor these patients with an annual ECG, and for patients in whom the ECG was initially normal we have recommended that an ECG be performed every three years. Furthermore, we recommend that medications that might cause QTc prolongation such as cisapride, antipsychotics (for example, thioridazine), tricyclic antidepressants, antiarrhythmics (such as quinidine, sotolol, and amiodarone), and antibiotics (such as erythromycin and ketoconazole) should be avoided in patients with a QTc abnormality. Long term follow up is required to interpret the reports of increased sudden death in patients with Rett syndrome and its possible association with QTc prolongation.

1 Clarke A. Rett syndrome. $\mathcal{F}$ Med Genet 1996;33:693-9.

2 Sekul EA, Moak JP, Schultz RJ, et al. Electrocardiographic findings in Rett syndrome: an explanation for sudden death? F Pediatr 1994;125:80-2.

3 Leonard H, Bower C, English D. The prevalence and Leonard H, Bower C, English D. The prevalence and
incidence of Rett syndrome in Australia. Eur Child Adolesc incidence of Rett syndrome in Aust
Psychiatry 1997;6:(suppl 1):8-10.

4 Trevathan E, Moser HV. Diagnostic criteria for Rett syndrome. Ann Neurol 1988:23:425-8.

5 Hagberg BA, Skejldal OH. Rett variants: a suggested model for inclusion criteria. Pediatr Neurol 1994;11:5-11.

6 Hagberg B, Witt Engerstrom I. Rett syndrome: a suggested staging system for describing impairment profile with increasing age towards adolescence. Am f Med Genet 1986; 24:47-59.

7 Bazett HC. An analysis of the time-relations of electrocardiograms. Heart 1920;7:353-70.

8 Leonard $\mathrm{H}$, Bower C. Is the girl with Rett syndrome normal at birth? Dev Med Child Neurol 1998:40:115-21.

9 Kerr AM, Armstrong DD, Prescott RJ, Doyle D, Kearney DL. Rett syndrome: analysis of deaths in the British survey. DL. Rett syndrome: analysis of deaths in the British

10 Bailey BP. The long QT syndromes. Aust N Z F Med 1996; 26:834-40.

11 Galloway PA, Glass PS. Anesthetic implications of prolonged QT interval syndromes. Anesth Analg 1984:64:61220.

2 Wang Q, Curran ME, Spawski I, et al. Positional cloning of a novel potassium channel gene: KVLQT1 mutations cause cardiac arrhythmias. Nat Genet 1996;12:17-23.

13 Towbin JA, Freidman RA. Prolongation of the QT interval and the sudden infant death syndrome. $N$ Engl $f \mathrm{Med}$ 1998;338:1760-1. 